\title{
Characterisation and Application of Idle Period Durations in IEEE 802.11 DCF-based Multihop Wireless Networks
}

\author{
Van Nam Nguyen \\ Université Claude Bernard \\ Lyon 1 - IFI \\ IRD, UMI 209 UMMISCO, MSI \\ Team \\ LIP (UMR ENS Lyon - INRIA - \\ CNRS - UCBL) \\ Lyon, France \\ nguyen.van.nam@ens- \\ lyon.fr
}

\author{
Isabelle Guérin Lassous \\ Université Claude Bernard \\ Lyon 1 \\ LIP (UMR ENS Lyon - INRIA - \\ CNRS - UCBL) \\ Lyon, France \\ isabelle.guerin- \\ lassous@ens-lyon.fr
}

\author{
Tahiry Razafindralambo \\ IRCICA/LIFL \\ Univ. Lille 1, CNRS UMR \\ 8022, INRIA LNE/ POPS \\ tahiry.razafindralambo@inria.fr
}

\author{
Victor Moraru \\ Institut de la Francophonie \\ pour l'Informatique (IFI) \\ IRD, UMI 209 UMMISCO, MSI \\ Team \\ Hanoi, Vietnam \\ victor.moraru@auf.org
}

\begin{abstract}
Multihop wireless networks are used to provide internet connectivity to the users and the level of performance and quality expected by these users are increasing. In order to meet these performance and quality requirements, wireless communications should be enhanced. Previous works from the literature show that the performance and quality provided by an IEEE 802.11-based multihop wireless network are far from optimal and that there exist different ways to increase the efficiency and the quality of service of such a network. Some studies show that using the medium state as a parameter to tune the behaviour of an IEEE 802.11-based multihop network is an appropriate way to proceed. A station in a IEEE 802.11-based multihop wireless network senses the medium either busy or idle. The durations of idle periods and busy periods and their distributions have a clear impact on the network and nodes performance. The understanding of the relationship between these indicators, namely idle and busy periods, the network topology and the traffic, would give new insights to enhance the performance and quality of multihop wireless networks. Due to its multihop and distributed nature, the characterisation of idle period durations is difficult in such a network. This paper explores the characterisation of idle period distribution by proposing a new analytical model and provides an application of this characterisation with the design of an adaptive backoff algorithm based on idle periods.
\end{abstract}

Permission to make digital or hard copies of all or part of this work for personal or classroom use is granted without fee provided that copies are not made or distributed for profit or commercial advantage and that copies bear this notice and the full citation on the first page. To copy otherwise, to republish, to post on servers or to redistribute to lists, requires prior specific permission and/or a fee.

MSWiM'12, October 21-25, 2012, Paphos, Cyprus.

Copyright 2012 ACM 978-1-4503-1628-6/12/10 ...\$15.00.

\section{Categories and Subject Descriptors}

C.2.1 [Computer-Communication Networks]: Network Architecture and Design-Wireless Communication

\section{General Terms}

Measurement, Performance

\section{Keywords}

Idle Periods, Multihop wireless networks, IEEE 802.11, Quality of service

\section{INTRODUCTION}

A station in a IEEE 802.11-based multihop wireless network senses the medium either busy or idle. The durations of idle periods and busy periods have a clear impact on the network and nodes performance. The characterisation of idle period durations is an important issue in wireless and mobile networks. For example, in cognitive radio networks, a secondary node must be able to adapt its behavior based on observations of the radio medium and on its experience in the past. The cognitive process is composed of the analysis of conditions observed on the network, of the decisionmaking and of the learning based on its experience. Under these conditions, the observation and the analysis of idle period durations and their statistical parameters are important for the proper functioning of this approach [9]. It is also possible to rely on observed idle periods to estimate the number of active nodes in the network, when all nodes are in the same carrier sense area [2]. Observation of idle period durations are also used in QoS solutions, like in [10] that describes an available bandwidth estimation between two nodes based on the nodes' average idle time durations. It is also the authors point of view that it should be possible to improve efficiency and fairness of MAC solutions for multihop wireless networks thanks to a knowledge of idle period durations. 
As far as we know, very few works have attempted to characterise idle period durations in multihop wireless networks. In [11], the authors characterise interarrival times (of TCP flows) on any node via an analytical model. The considered networks are WLANs with and without infrastructure. They show that interarrival times have a multimodal distribution. The proposed model could be used to infer idle time durations but the work assumes that there is no hidden node. In [3], the authors seek to characterize idle periods to estimate the number of nodes in competition on a CSMA/CA network. This work is valid for single-hop networks with nodes that have always a packet to send and that always use the same backoff stage (the initial one). In [9], the authors characterize idle period durations in 802.11e single-hop networks (based on EDCA) with Poisson traffic arriving at each node. They show that, when the traffic intensity is non negigible, the distribution of idle period durations can be approximated by a Gamma distribution. All these works are mainly focused on single-hop networks and do not consider multihop networks whose features impact differently idle periods.

In studies of multihop wireless networks, assumptions on idle periods are often considered. For instance, Garetto et al. [5] model the wireless channel as a stochastic process in which the durations of busy and idle periods of nodes are exponentially distributed. Kolar et al. [7] model the backoff and transmission periods as an Alternating Renewal Process in which backoff time and packet size are exponentially distributed. In [10], the authors assume that idle periods, for which the total duration is known (by measurement), are uniformly distributed in a given period of observation. As far as we know, there is no evidence that these assumptions are appropriate.

In this paper, we study the distribution of idle periods in multihop wireless networks based on IEEE 802.11. As opposed to the other existing works, we consider multihop networks with potential hidden stations and under nonnegligible traffic intensity. In this work, we first study the distribution of idle periods in a basic multihop configuration. Then, we propose an analytical model to characterise idle period durations in multihop wireless networks. Finally, we use the previous analysis to adress one problem in multihop wireless networks, namely the design of a fair MAC solution.

This article is organized as follows: in Section 2, we summarize the main principles of $802.11 \mathrm{DCF}$ and we give the main assumptions of our study. In Section 3, we study, by simulation, the distribution of idle periods in a basic multihop wireless scenario. Then, in Section 4, we propose an analytical model for the distribution of idle periods for a node in an arbitrary multihop toplogy. The model is validated with simulations in Section 5. In Section 6, we use the study of the idle period distribution, carried out in the previous sections, to propose a simple and fair MAC protocol for multihop wireless networks.

\section{802.11 DCF PRINCIPLES AND ASSUMP- TIONS}

As we consider 802.11-based multihop wireless networks, we first describe the main principles of the DCF mode of 802.11 that impact idle periods in the network. We then discuss the main assumptions we make for our study.

\subsection{DCF main principles}

IEEE 802.11 DCF [8] is widely used as the MAC layer of multihop wireless networks due to its distributed nature. In the DCF (Distributed Coordination Function) mechanism, a node that wants to transmit a packet has to wait for a fixed period DIFS and a random period, called backoff, randomly chosen in a contention window. During these periods, the medium must be idle. During the backoff counting process, if the medium is detected as busy, the backoff counter is frozen and will be resumed as soon as the medium returns to the idle state. As soon as the backoff counter reaches zero and if the channel is idle, the node starts the transmission of its packet. In $802.11 \mathrm{DCF}$, a unicast packet is considered to be successfully transmitted if the sender receives an acknowledgement from the receiver after the fixed period SIFS. Otherwise, the sender considers that a collision has occurred. The packet is then retransmitted by using the same process but with a contention window size that is doubled. After each successive collision, the contention window size is doubled (the contention window is also called the backoff stage and the stage number depends on the number of successive undergone collisions). The number of packet retransmissions is limited to a maximal value defined by the standard. The value of the backoff stage is set to its initial value if the transmission succeeds or if the packet is dropped after a maximal number of retransmissions without success.

\subsection{Assumptions}

In this article, we assume that the RTS/CTS mechanism is disabled. We also assume that the packet size, denoted by $T$ hereafter, is fixed and expressed in number of slots, that the used modulation is fixed and that the physical layer is perfect (i.e. there is no packet loss due to physical error). We do not consider SIFS time between a packet and its MAC acknowlegment like an idle period since it is part of an entire 802.11 transmission. In our study, when a backoff will be considered, we will assume that the DIFS time is included since a backoff is always associated to a DIFS.

Finally, we consider that an idle period perceived by a node is the time during which no signal is sensed on the wireless channel with a power higher than the carrier sense threshold. ${ }^{1}$

\section{FIRST STUDY: A BASIC SCENARIO}

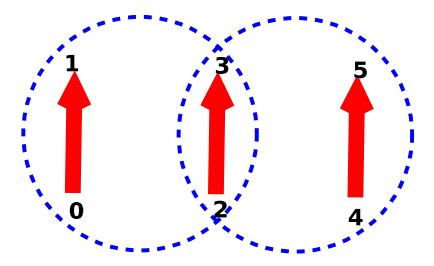

Figure 1: Flow In the Middle (FIM) scenario

In this section, we study, by simulation, the distribution of idle periods in the basic Flow in the Middle (FIM) scenario (Fig. 1) [4]. The scenario consists of six nodes and three flows. The nodes $(0,1,2,3)$ are in the same sensing range,

\footnotetext{
${ }^{1}$ Note that other mechanisms exist to determine whether the medium is idle; this is called the Clear Channel Assessment process.
} 


\begin{tabular}{|l|c|}
\hline Propagation model & 2-Ray Ground \\
\hline SIFS & $10 \mu \mathrm{s}$ \\
\hline DIFS & $50 \mu \mathrm{s}$ \\
\hline Backoff time slot & $20 \mu \mathrm{s}$ \\
\hline Transmision range & $200 \mathrm{~m}$ \\
\hline Carrier sensing range & $250 \mathrm{~m}\left(^{2}\right)$ \\
\hline Physical rate & $2 \mathrm{Mb} / \mathrm{s}$ \\
\hline Flows & $1 \mathrm{kbyte}$ \\
\hline Packet size size & 32,1024 \\
\hline Retransmision limit & $\mathrm{ASR}\left({ }^{3}\right)$ \\
\hline $\begin{array}{l}\text { Contention window } \\
\text { (min, max) }\end{array}$ & $100 \mathrm{~s}$ \\
\hline Routing Protocol & \\
\hline Simulation time &
\end{tabular}

Table 1: Nodes configuration with Ns2.33

like the nodes $(2,3,4,5)$, while the nodes $(0,1)$ are hidden to the nodes $(4,5)$ (and vice versa). In the simulations carried out with Ns2.33, all nodes are configured according to the parameters given in Table 1.

The distribution of idle periods of nodes are depicted in Figs. 2 and 3. In Fig. 2, the interior figure is a focus on the part of the distribution around $5000 \mu \mathrm{s}$. From these figures, we find that the nodes $(0,1,4,5)$ have identical bimodal distributions and that Nodes 2 and 3 have also the same mono-modal distribution.

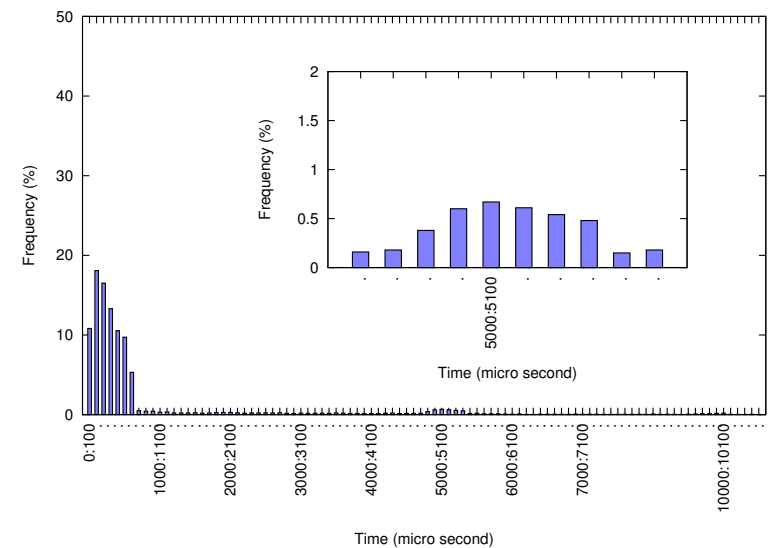

Figure 2: Distribution of idle period durations for the nodes $(0,1,4,5)$ in the FIM scenario

We identify three main kinds of idle periods in this scenario:

- Entire backoff time (denoted as B): In IEEE 802.11 DCF, a random backoff time is always associated to a packet transmission. If the backoff counter is not interrupted, then, for each backoff stage, the backoff time is uniformly distributed in the associated contention window. In the tested FIM scenario, collisions

\footnotetext{
${ }^{2}$ This range may be low compared to reality, but with this value, we easily simulate hidden nodes.

${ }^{3}$ To ensure that no routing control packet is sent and competes with data packets, we use a static routing protocol (called ASR). It simplifies the analysis but not the shape of the distribution.
}

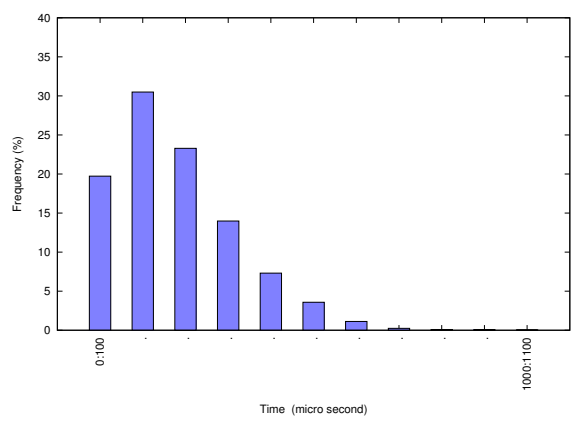

Figure 3: Distribution of idle period durations for the nodes $(2,3)$ in the FIM scenario

are only possible if the backoff counters of two (or more) sources in the same sensing range reach zero at the same time. This happens very rarely. Therefore, the sources mainly draw their backoff in the minimum contention window. We notice that the first peak of each distribution ${ }^{4}$ is mostly included in the interval $[0: 620 \mu \mathrm{s}]$ which corresponds to the minimum contention window size. Thus, this peak represents backoff time and idle periods corresponding to entire backoff times are included in this first peak. However, the first peak is not uniform, which means that the nodes' backoff counter is sometimes interrupted.

- Interrupted backoff time (denoted as $\breve{B}$ ): When the backoff counter of a node is interrupted, the backoff time is separated into several smaller periods. These idle periods are also included in the first peak of the distribution of the six nodes. Depending on their neighbors' activity in terms of packets to send, the nodes have different frequencies of backoff interruptions. For instance, Node 2 is impacted by the packet transmissions of five nodes $(0,1,3,4,5)$, while Node 0 is only impacted by the packet transmissions of three nodes $(1,2,3)$. Therefore, the frequency of backoff interruptions of Node 2 is greater than the one of Node 0 , which explains that Node 2 has smaller idle periods in its distribution.

- Backoff and interruption time (denoted as $\widehat{B}$ ): In the tested FIM scenario with TCP flows, nodes have sometimes no packet to send at the MAC level while neighbors in the same sensing range have packets to transmit. However, these nodes sense the medium idle during a period larger than a backoff. This fact can be explained by the transmissions of nodes that are hidden to nodes that have no packet to send and that freeze the backoff counter of the neighbor nodes. For instance, in the FIM scenario, when the backoff counter of Node 2 is interrupted by a packet transmission of Node 4, the backoff of Node 2 is frozen during a packet transmission. During this period, Node 0, that has no packet to send, considers that the channel is idle during a period larger than a backoff. Such a phenomenon is depicted on Figure 4. The second peak of the idle period distribution for the nodes $(0,1,4,5)$ corresponds to this kind of idle period. As with TCP

\footnotetext{
${ }^{4}$ that is the single peak for Nodes 2 and 3.
} 
flows, the probability that the packets interarrival time on the MAC level of a node is greater than zero is non-negligible, it explains why the idle periods due to backoff and interruptions of hidden nodes exist in this scenario even if their frequency is small.

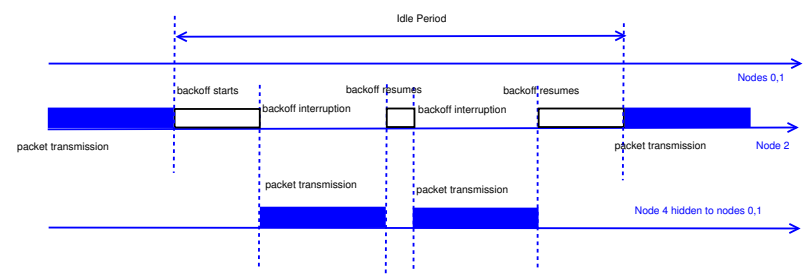

Figure 4: Idle period due to backoff and interruptions from hidden nodes

From the previous observations, we see that three kinds of idle period impact the idle period distribution of nodes in the FIM scenario: the entire backoff times, the interrupted backoff times and the backoff and interruption times from hidden nodes. The entire backoff time depends on the backoff stage in which the backoff is drawn. The backoff stage depends on the collision probability. Therefore, the collision probability impacts the width of the peaks of the distribution. The interrupted backoff depends on the activity of the neighbor nodes in the same sensing range. Therefore, the packet transmission rate of neighbor nodes impacts the height of the peaks of the distribution. The backoff and interruption time depends on the number of hidden nodes and their activity. Therefore, the number of hidden nodes impact the number of peaks in the distribution and the packet transmission rate of hidden nodes impact the width of the distribution.

\section{ANALYTICAL MODEL}

In this section, we propose an analytical model, based on the three kinds of idle periods identified previously, in order to compute the distribution of idle periods of a node in an arbitrary multihop topology.

\subsection{Model assumptions}

In this paper, we assume that the traffic pattern transmitted by the nodes in the multihop wireless network is stochastic with a non-negligible traffic intensity. With this assumption, the probability that a node has packets to send is non-negligible and the probability that the wireless channel be saturated is also non-negligible. We also assume that the hidden stations (as defined in Section 4.2) of a same node have independent activities.

\subsection{System Model}

We model the wireless channel of a given node in a multihop wireless network as follows. Given a node $A$, the wireless channel of $A$ is impacted by the nodes which are in the same sensing range, like $B, C$ and $D$ (see Fig. 5 ; the dotted circle represents the carrier sensing area of $A$ ). The wireless channel of $A$ is also impacted by hidden nodes of $A$ which are out of its sensing range (like $E, F$ and $G$ in Fig. 5), but which impact the behavior of nodes in the carrier sensing area of $A$. Note that, here, the term hidden nodes has a large meaning. Indeed, it corresponds to nodes that can not be sensed by Node $A$ but these nodes' activity may provoke collisions on packets transmitted in the carrier sensing area of $A$ or may prevent a node in the carrier sensing area of $A$ from transmitting.

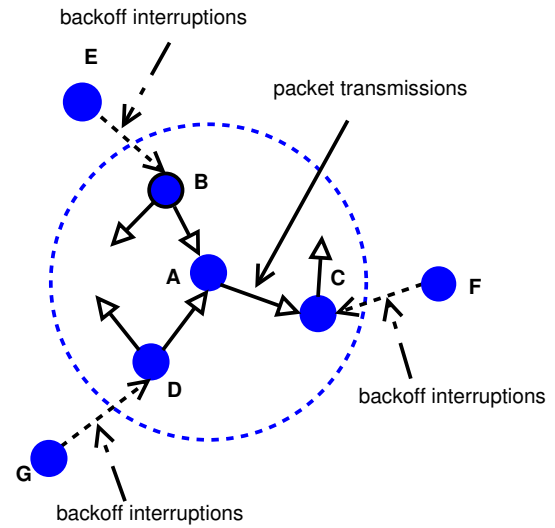

Figure 5: System model for the wireless channel of a node

These impacts are represented by the following parameters:

- Packet transmission rate $(\lambda)$ : This is the average packet output rate at the MAC level from all the nodes which are in the sensing range of $A$ (including $A$ ).

- Hidden transmission rate $\left(\lambda_{h}\right)$ : This is the average packet output rate at the MAC level from hidden nodes of $A$. These hidden nodes are in the sensing range of active neighbors of $A$.

- Collision probability $(p)$ : This is the average collision probability for a packet transmitted in the carrier sensing area of $A$.

\subsection{Distribution of entire backoff times}

First, we determine the idle periods of Node $A$ due to entire (not interrupted) backoff drawn by $A$ or by nodes in the carrier sensing area of $A$. Given a contention window, the drawn backoff is uniformly distributed in this window. Once a packet has collided, it will be retransmitted with a contention window whose size is doubled. The backoff time depends therefore on the number of retransmissions.

Given the collision probability $p$, the probability mass function for the number of retransmissions per packet [1] is:

$$
\operatorname{Pr}(n=i)= \begin{cases}p^{i} *(1-p), & 0 \leq i<M \\ p^{M}, & i=M\end{cases}
$$

where $M$ is the retransmission limit.

Let's define $N$ such that $2^{N} * C W_{\min }$ is the maximal contention window size $\left(C W_{\max }\right)$. For a value $t$ (expressed in number of slots), we denote $m=\left|\log _{2}\left(\frac{t}{C W_{\min }}\right)\right|$. The probability that the backoff is equal to $t$ can then be expressed as follows: 
- If $N<M-1$ :

$$
P(B=t)=\left\{\begin{array}{l}
\sum_{i=m}^{N} \operatorname{Pr}(n=i) * \frac{1}{2^{i} * C W_{\min }}, 0 \leq m \leq N \\
\sum_{i=m}^{N} \operatorname{Pr}(n=i) * \frac{1}{2^{i} * C W_{\min }}+\frac{m-N}{2^{N} * C W_{\min }}, \\
N<m \leq M-1 \\
0, \text { otherwise }
\end{array}\right.
$$

- If $M-1 \leq N$ :

$$
P(B=t)=\left\{\begin{array}{l}
\sum_{i=m}^{M-1} \operatorname{Pr}(n=i) * \frac{1}{2^{i} * C W_{\min }}, \\
0 \leq m \leq M-1 \\
0, \text { otherwise }
\end{array}\right.
$$

\subsection{Distribution of interrupted backoff times}

In this section, we determine the idle periods of Node $A$ that correspond to backoff interrupted by the transmissions of $A$ or by nodes in the carrier sensing area of $A$. In [9], the authors show that the interrupted backoff times (including also entire backoff times) follow a Gamma distribution. The probability that the interrupted backoff time is equal to $t$ (expressed in number of slots) can be expressed as follows:

$$
\operatorname{Pr}(\breve{B}=t)=\frac{\beta^{\alpha}}{\Gamma(\alpha)} t^{\alpha-1} e^{-\beta t}
$$

Given the mean $E(\breve{B})$ and variance $\operatorname{Var}(\breve{B})$, the parameters $\alpha$ and $\beta$ can be approximated as follows:

$$
\begin{gathered}
\beta=\frac{E(\breve{B})}{\operatorname{Var}(\breve{B})} \\
\alpha=E(\breve{B}) \beta
\end{gathered}
$$

Note that, the interrupted backoff times are caused by the packet transmission rate $(\lambda)$ and the sizes of the associated contention windows to transmit these packets $(C W)$. Both $E(\breve{B})$ and $\operatorname{Var}(\breve{B})$ can therefore be calculated from $\lambda$ and $C W: E(\breve{B})$ can be estimated as $\frac{1}{\lambda}$ and $\operatorname{Var}(\breve{B})$ can then be derived from $E(\breve{B})$ and $C W$ by using the formulas in [6].

\subsection{Distribution of backoff and interruption times}

In this part, we determine the idle periods of Node $A$ larger than a backoff and that are due to an empty queue at the MAC level for Node $A$ and transmissions of nodes hidden to $A$ that prevent nodes in the carrier sensing area of $A$ from transmitting. Since we assume that hidden nodes of a same node have independent activities, then we consider that the packet arrival from these hidden nodes follows a Poisson distribution. If there are $k$ arrivals during an entire backoff time $B$, then the interruption time due to packet transmissions can be expressed as follows:

$$
S^{k}=k * T
$$

where $T$ is the packet transmission time. The values of $S^{k}$ are discrete and multiple of $T$.

The probability that $S^{k}$ is equal to $t$ (expressed in number of slots) can be estimated as follows:

$$
\operatorname{Pr}\left(S^{k}=t\right)=\left(\lambda_{h} * T\right)^{k} * \frac{e^{-\lambda_{h} * T}}{k !} * \operatorname{Pr}(k * T=t)
$$

The idle periods due to backoff and interruptions from hidden nodes can be expressed as follows:

$$
\widehat{B}=B+S^{k}
$$

Given the distributions of $B$ and $S^{k}$, we can derive the distribution $\widehat{B}$ by the convolution approach.

Since the difference between two consecutive values of $B$ is much smaller than the difference between two consecutive values of $S^{k}$, the distribution of $\widehat{B}$ is multimodal. Moreover, the difference between two consecutive peaks is equal to a packet transmission time $T$.

\subsection{General distribution of idle periods}

Finally, we determine the general distribution for the idle periods of Node $A$. Given the output rate $\lambda$ in the wireless channel of Node $A$ and the packet transmission time $T$, the offered load of the wireless channel of Node $A$ is $\rho=\lambda * T$. As a packet transmission is always associated to a backoff time, the probability that the idle periods of Node $A$ corresponds to entire and interrupted backoff times is then $\rho$. The probability of other kinds of idle periods is then $1-\rho$. Therefore, we estimate the distribution of idle periods (denoted $I P$ ) of Node $A$ as follows:

$$
\operatorname{Pr}(I P=t)=\rho * \operatorname{Pr}(\breve{B}=t)+(1-\rho) * \operatorname{Pr}(\widehat{B}=t)
$$

$(1-\rho) * \operatorname{Pr}(\widehat{B}=t)$ corresponds to the probability that nodes in the carrier sensing range of $A$ be blocked by transmissions of hidden nodes of $A$ and that $A$ has no packet to send.

\subsection{Discussion}

The distribution of idle periods given in Equation 10 reflects what we have analyzed with the FIM scenario (Section 3). The contention window size determines the width of the distributions of $\breve{B}$ and $B$ and impacts the width of the distribution $\widehat{B}$. Since there is only one part in the distribution of $\breve{B}$, the number of peaks of the general idle period distribution is the number of peaks in the distribution of $\widehat{B}$ plus one. These minor peaks are due to hidden nodes.

Since we consider networks under non-negligible traffic intensity, $\rho$ is rather high. The height of the distribution of $\breve{B}$ will determine the height of the general idle period distribution.

\section{MODEL VALIDATION}

In this section, we validate our proposed model by simulations with Ns2.33.

\subsection{Topologies}

We generate two random topologies on an area of $(1000 \mathrm{~m}, 1000 \mathrm{~m})$. The first one (called 20/47 topology) consists of 20 nodes and 47 single-hop TCP flows. The second one (called 100/176 topology) includes 100 nodes and 176 single-hop TCP flows. The nodes are now configured with more realistic communication range $(250 \mathrm{~m})$ and sensing range $(550 \mathrm{~m}$ ) (all the other parameters of Ns2.33 are identical to the ones given in Table 1).

Then, we randomly select two nodes in each topology and we compare, for each node, the idle period distribution obtained by simulation and the one computed with our analytical model. Note that, with our model, we use simulation 
results to compute $\lambda, \lambda_{h}, p, E(\breve{B})$ and $\operatorname{Var}(\breve{B})$ required for the computation of $\operatorname{Pr}(\breve{B}=t)$, rather than general formulas.

\subsection{Simulation Results}

The distributions of idle periods of Nodes $0(39 m, 546 m)$ and $5(570 m, 525 m)$ of the 20/47 topology are given in Figs. 6 and 7 . The interior figures show the different parts of the distribution other than the first peak.

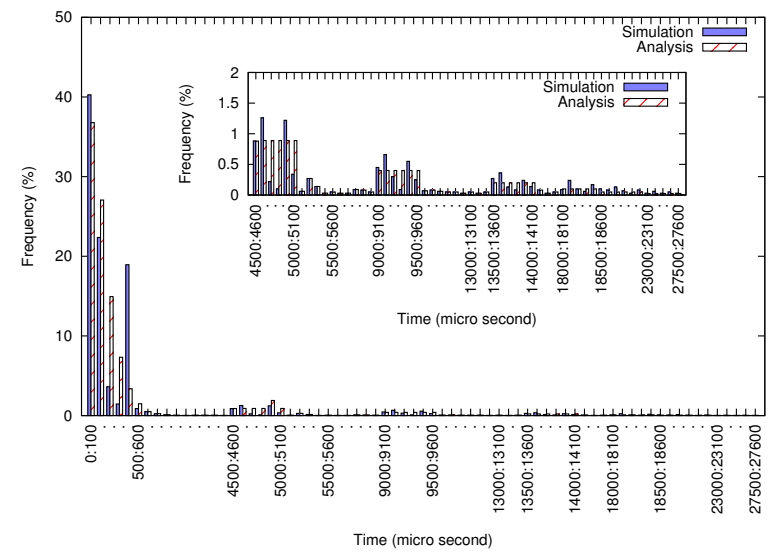

Figure 6: Distribution of idle periods of Node 0 in the $20 / 47$ topology

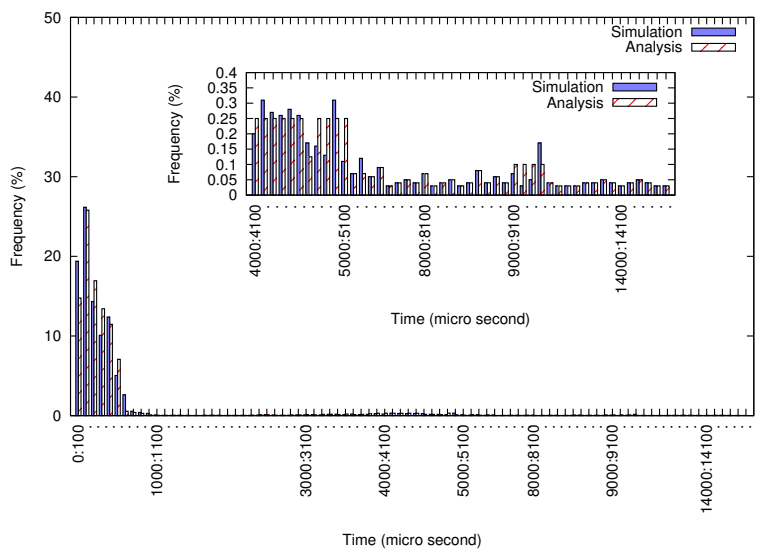

Figure 7: Distribution of idle periods of Node 5 in 20/47 topology

First, we see that the results computed from our proposed model are close to the ones obtained by simulation. From these two figures, we find that the two nodes have the first part of their distribution in the range $([0: 600] \mu s$ approximately). It corresponds to backoff times (interrupted or not). This part represents more than $90 \%$ of the idle periods. The same kind of result is observed on the other nodes of the network.

However, these distributions have other parts that are smaller (in terms of frequency) than the first part. When we plot them in another scale, we clearly see that the distributions are multimodal. The peaks of these distributions are multiple of $4500 \mu \mathrm{s}$ which is the transmission time for a packet of 1 kbyte transmitted at $2 \mathrm{Mb} / \mathrm{s}$. These parts correspond to idle periods due to backoff and interruptions of hidden nodes. There are five peaks in the distribution of Node 0 (at $4500 \mu s, 9000 \mu s, 13500 \mu s, 18000 \mu s, 22500 \mu s$ ), while there are only three peaks in the distribution of Node 5 (at $4500 \mu s, 9000 \mu s, 13500 \mu s$ ).

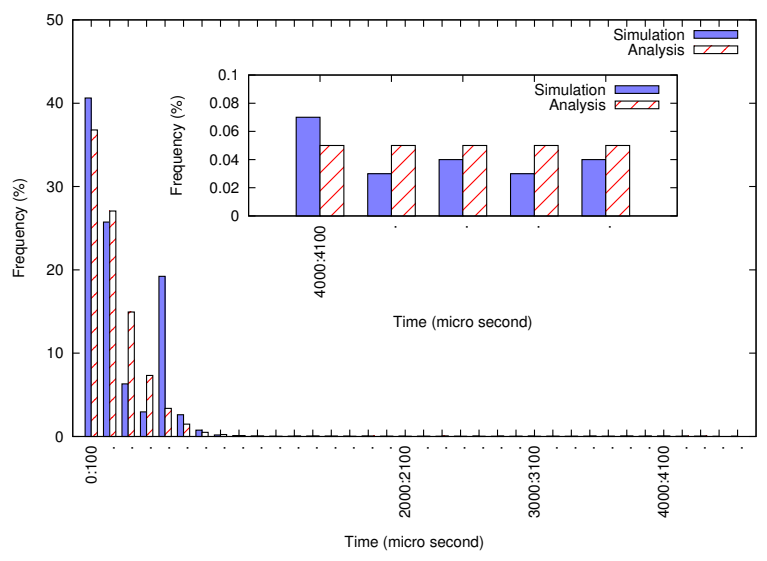

Figure 8: Distribution of idle periods of Node 61 in the $100 / 176$ topology

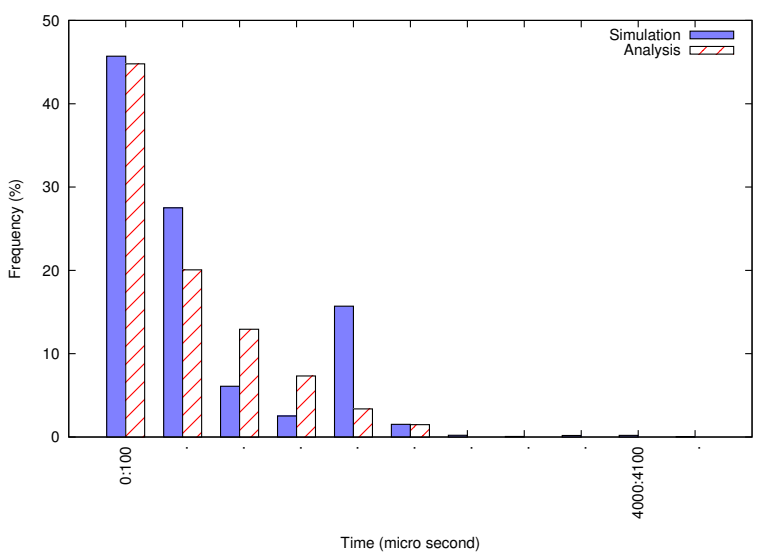

Figure 9: Distribution of idle periods of Node 50 in 100/176 topology

Simulation results for the idle period distributions of Nodes $61(64 m, 756 m)$ and $50(180 m, 726 m)$ of the $100 / 76$ topology are given in Figs. 8 and 9. In the distribution of Node 50, there is only one peak that corresponds to backoff interruptions, while there are two parts in the distribution of Node 61. As the 100/176 topology is very loaded, the frequency of idle periods due to backoff and interruptions of hidden nodes is smaller than with the 20/47 topology.

These differents results, observed on the selected nodes, but also on the other nodes, show that the first parts of the distributions are relatively similar between the nodes, while the other parts may be very different from one node to another. These parts are correlated to the number of hidden nodes and the way these hidden nodes are located in the topology (relative to the neighbors and to the other hidden nodes). This can be very different from node to node and from topology to topology. 


\section{APPLICATION: IDLE PERIOD-BASED ADAP- TIVE BACKOFF SCHEME (IABS)}

In this section, we discuss a possible use of idle periods and their distribution in multihop wireless networks under non-negligible traffic intensity. We propose a simple backoff algorithm, called idle period-based adaptive backoff scheme (iABS), to solve the unfairness phenomenon that arises in multihop wireless networks. This scheme uses the backoff and interruption times from hidden nodes observed on minor peaks of the idle period distribution and the neighbors' activity.

We define $C X$, the contention index of a node as:

$$
C X=\left\lfloor\frac{\widehat{B}_{\text {sum }}}{L_{\text {arrival }}}\right\rfloor
$$

where $\widehat{B}_{\text {sum }}$ is the total backoff and interruption times (expressed in the number of slots) of the node during an observation period (and extracted from the secondary peaks in the idle period distribution) and $L_{\text {arrival }}$ is the total data packet arrivals on the radio medium from neighboring nodes. $C X$ is close to zero if the hidden nodes' activity is much less important than the neighbors' activity. $C X$ is large if the hidden nodes' activity is much more important than the neighbors' activity.

iABS operates as follows: upon reception of an acknowledgement, a node modifies its minimum contention window $C W_{\min }$ for the new packet to send as follows:

$$
C W_{\min }^{\text {new }}=C W_{\text {min }}^{\text {original }}+\omega * C X^{\text {current }}
$$

where $\omega$ is a scaling factor which is constant for each topology, $C W_{\text {min }}^{\text {original }}$ is the defaut value of $C W_{\min }$ and $C X^{\text {current }}$ is the contention index of the node during the current observation period. Note that, at the end of an observation period, we set $C W_{\min }$ to its default value $C W_{\text {min }}^{\text {original }}$. It ensures that $C W_{\min }$ does not increase to a large value through time.

With this algorithm, the backoff time of a node is increased according to its contention index. If its contention index is high, it means that hidden nodes have much activity than its neighbors. Therefore, the minimal contention window size is increased in order to reduce the channel access probability of the node and to increase the one of its neighbors. If its contention index is low, it means that its neighbors have much activity. Therefore, the minimal contention window size is decreased in order to increase the channel access probability of the node and to decrease the one of its neighbors. The goal of the adjustment of the minimal contention window size is to make the contention indexes of the nodes in competition in the wireless channel access converge to a same value.

We have evaluated iABS with Ns2.33. The communication and sensing ranges of the nodes are $250 \mathrm{~m}$ and $550 \mathrm{~m}$, respectively (the other parameters are given in Table 1). Fig. 10 shows the results obtained on the FIM scenario with and without iABS. We see that, with iABS, the flow rates converge quickly to the same rate and that the unfairness issue raised by this topology is solved.

We have also tested iABS on three random topologies which are generated in an area of $(1000 \mathrm{~m}, 1000 \mathrm{~m})$. The first topology consists of 10 nodes and 14 TCP one-hop flows (and is denoted 10/14 topology). The second one consists of 30 nodes and 37 TCP one-hop flows, while the third topology

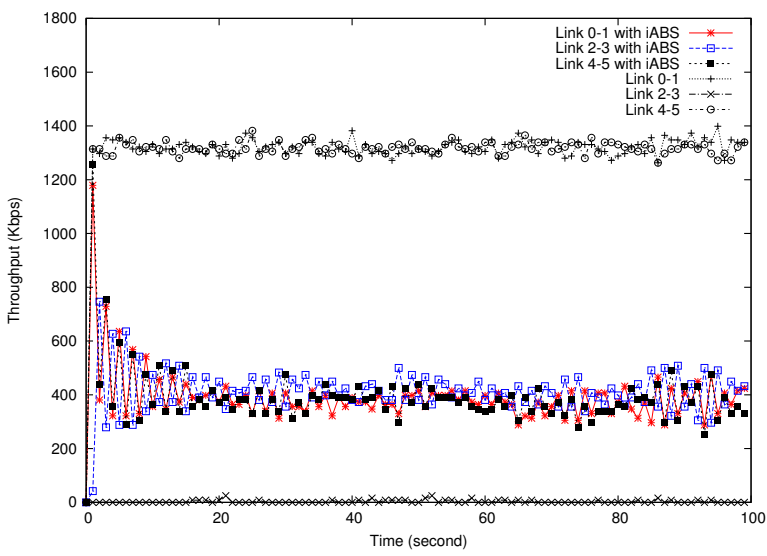

Figure 10: iABS for the FIM scenario

includes 50 nodes and 97 TCP one-hop flows. In the 50/97 topology, we randomly select three TCP flows: Flow 0 (from Node $0(811 m, 446 m)$ to Node $24(828 m, 489 m))$, Flow 50 (from Node $3(390 m, 377 m)$ to Node $49(349 m, 396 m)$ ) and Flow 90 (from Node 49 to Node $9(158 m, 426 m)$ ). Fig. 11 shows the rate of these three flows without and with the use of iABS. We see that the use of iABS provides fairer results than 802.11 DCF. This observation is also confirmed by the results provided in Fig. 12 that shows the Jain fairness index for the three tested topologies. An index close to 0 indicates an unfair situation, while an index close to 1 means that flows obtained similar throughputs. From this figure, we see that iABS reduces the unfairness issue on the three topologies, specifically on the $30 / 37$ and $50 / 97$ topologies.

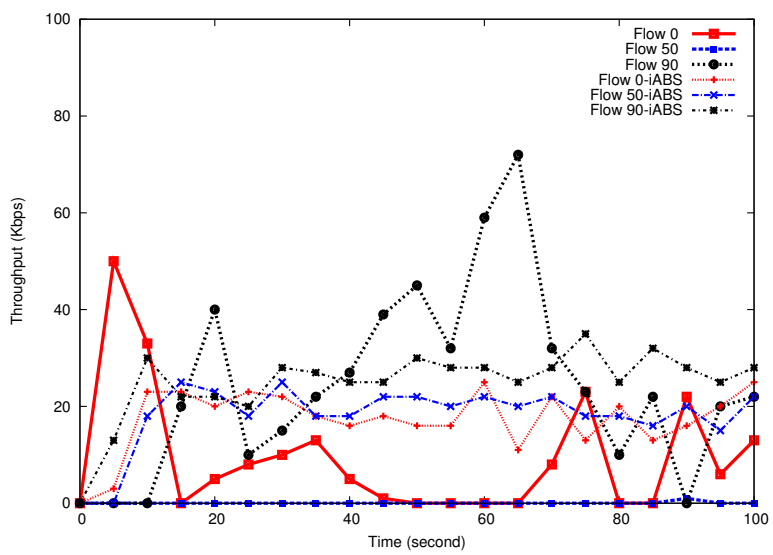

Figure 11: The rates of flows with the use of iABS in the $50 / 97$ topology

On these preliminary tests, we can conclude that iABS provides very efficient results in terms of fairness without message overhead since iABS uses only the idle period distribution (and more precisely the secondary peaks induced by hidden nodes) and the neighbors' activity.

\section{CONCLUSION}

In this paper, we propose a novel analytical model for the distribution of idle periods of nodes in relatively saturated 802.11-based multihop wireless networks. Most of 


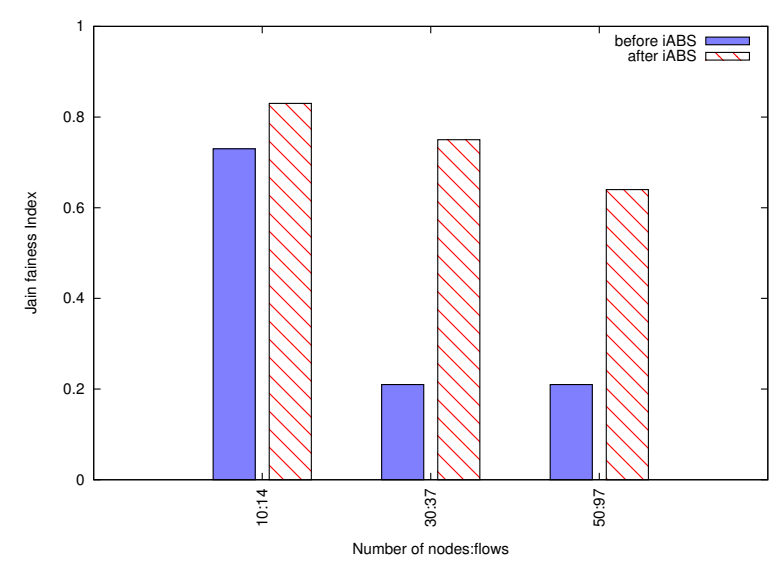

Figure 12: Jain fairness index obtained on the three topologies with and without iABS

propoposed models in literature mainly consider common ilde periods of the nodes such as the backoff times. Our model takes into account longer idle periods, called backoff and interruption times and induced by hidden nodes. This kind of idle periods can be very different from one node to another. The distribution of idle periods, provided by our model, is therefore characterized by the number of peaks rather than the frequency of idle periods. The validation in random and large topologies shows that our analytical model matches well the simulation results in Ns2.33.

Based on the analytical model and its analysis, we also propose a simple and efficient adaptive backoff algorithm to solve the contention unfairness in these networks. This algorithm relies on local information and has no communication overhead. It enables an equalization not only of the neighbors' activities but also those of hidden nodes. The Jain fairness index obtained from simulations in Ns2.33 in random and large topologies proves the efficiency and the simplicity of the algorithm.

The proposed analytical model is extensible for different packet sizes and for unsaturated wireless networks. The different packet sizes may impact the distance between the peaks. Moreover, they also imply changes into the packet collisions and therefore the width of the peaks in the distribution. In unsaturared networks, there may exist other kinds of idle periods such as the residual interarrival times. If the interarrival times are continuous then there is no more new peak in the distribution. It may be more difficult to distinguish the peaks. However, the general form of the distribution remains unchanged. It is also possible to take into account errors from the physical layer and/or the different modulations by adjusting the dynamic paramerers used in our model.

In future work, we plan to use the propeties of the idle period distribution characterized by the proposed analytical model in some solutions, like, for instance, the available bandwidth estimation. For instance, neighbor nodes could exchange the number of peaks of their distribution, rather than the whole distribution parameters, and each node could try to infer the multihop topology (like the number of hidden nodes) in which it is located based on this information. Such exchanges are easy to do compared to exchanges of the whole distribution and reduce the communication overhead.
We also plan to compare the performance of our proposed solution iABS with existing algorithms.

\section{Acknowledgments}

This work is partially funded by the French National Research Agency (ANR) under the project ANR VERSO RESCUE (ANR-10-VERS-003)

\section{REFERENCES}

[1] G. Bianchi. Performance analysis of the ieee 802.11 distributed coordination function. Selected Areas in Communications, IEEE Journal on, 18(3):535-547, 2000.

[2] R. Bowden and A. Coyle. Idle times analysis in a csma/ca network. In Proceedings of the 15th IEEE International Conference on Networks, ICON 200\%, 19-21 November 2007, Adelaide, Australia, pages 443-448. IEEE, 2007.

[3] R. Bowden and A. Coyle. Idle times analysis in a csma/ca network. In ICON'07, pages 443-448, 2007.

[4] C. Chaudet, I. Guérin Lassous, E. Thierry, and B. Gaujal. Study of the impact of asymmetry and carrier sense mechanism in IEEE 802.11 multi-hops networks through a basic case. In ACM Workshop on Performance Evaluation of Wireless Ad Hoc, Sensor, and Ubiquitous Networks (PE-WASUN'04), Venice, Italy, Oct. 2004.

[5] M. Garetto, T. Salonidis, and E. W. Knightly. Modeling per-flow throughput and capturing starvation in csma multi-hop wireless networks. In In Proc. of IEEE Infocom, 2006.

[6] S. Hozo, B. Djulbegovic, and I. Hozo. Estimating the mean and variance from the median, range, and the size of a sample. BMC Medical Research Methodology, $5(1): 13+, 2005$.

[7] V. Kolar, K. Bharath, N. B. Abu-Ghazaleh, and J. Riihijarvi. Contention in multi-hop wireless networks: model and fairness analysis. In Proceedings of the 12th ACM international conference on Modeling, analysis and simulation of wireless and mobile systems, MSWiM '09, pages 21-29, New York, NY, USA, 2009. ACM.

[8] L.S.Committee. ANSI/IEEE Std 802.11:Wireless LAN Medium Access Control(MAC) and Physical Layer (PHY) Specifications. IEEE Computer Society, 1999.

[9] J. V. Misic and V. B. Misic. Characterization of idle periods in ieee 802.11 e networks. In $W C N C$, pages 1004-1009. IEEE, 2011.

[10] C. Sarr, C. Chaudet, G. Chelius, and I. Guérin Lassous. Bandwidth Estimation for IEEE 802.11-Based Ad Hoc Networks. IEEE Transactions on Mobile Computing, 7(10):1228-1241, 2008.

[11] O. Tickoo and B. Sikdar. On the impact of ieee 802.11 mac on traffic characteristics. IEEE Journal on Selected Areas in Communications, 21(2):189-203, 2003. 